EPSC Abstracts

Vol. 14, EPSC2020-475, 2020

https://doi.org/10.5194/epsc2020-475

Europlanet Science Congress 2020

(C) Author(s) 2021. This work is distributed under

the Creative Commons Attribution 4.0 License.

\title{
Change detection analyses using simulated and actual ExoMars TGO-CaSSIS images: A case study based on past and present Gasa Crater gully activity
}

\author{
Vidhya Ganesh Rangarajan ${ }^{1}$, Livio L. Tornabene ${ }^{1}$, Gordon R. Osinski ${ }^{1}$, Frank P. Seelos ${ }^{2}$, Susan J. \\ Conway $^{3}$, Manish R. Patel ${ }^{4}$, Nicolas Thomas ${ }^{5}$, Gabriele Cremonese ${ }^{6}$, Maurizio Pajola ${ }^{6}$, Giovanni \\ Munaretto $^{6,7}$, Alice Lucchetti ${ }^{6}$, and the CaSSIS Team \\ ${ }^{1}$ Institute for Earth and Space Exploration/ Department of Earth Sciences, University of Western Ontario, London, ON, Canada \\ (vrangara@uwo.ca) \\ ${ }^{2}$ Applied Physics Laboratory, John Hopkins University, Laurel, MD, USA \\ ${ }^{3}$ CNRS Laboratoire de Planétologie et Géodynamique de Nantes, Université de Nantes, 2 rue de la Houssiniére, 44322, Nantes, \\ France \\ ${ }^{4}$ School of Physical Sciences, STEM, The Open University, Milton Keynes, UK \\ ${ }^{5}$ Physikalisches Institut, University of Bern, Sidlerstr. 5, 3012 Bern, Switzerland \\ ${ }^{6}$ INAF-Osservatorio Astronomico di Padova, Padova, Italy \\ ${ }^{7}$ Department of Physics and Astronomy, University of Padova, Padova, Italy
}

Introduction: The Martian surface hosts a variety of active surface processes [1-3] whose regular monitoring is key to providing us insights into past and present-day surface, geologic and climatic conditions [4]. Most change detection studies on Mars utilize time-series image acquisitions from the Mars Reconnaissance Orbiter (MRO) High Resolution Imaging Science Experiment (HiRISE; 25-50 $\mathrm{cm} / \mathrm{px}$ ) [5] and the Context Camera (CTX; 5-6 m/px) [6]. However, the relatively narrow HiRISE colour swath ( $20 \%$ of the image swath) results in a lower probability of observing surface changes with multiple wavelengths.

The Colour and Stereo Surface Imaging System (CaSSIS) onboard the ExoMars Trace Gas Orbiter (TGO) [7] permits 4-band VNIR colour coverage at $4.6 \mathrm{~m} / \mathrm{px}$ and an image swath $>6 \mathrm{~km}$. Furthermore, TGO/CaSSIS is able to observe Mars at multiple times of day, permitting detection/monitoring of diurnal processes.

While TGO has only been in operation for a short period of time (MY34-35), the development of simulated CaSSIS images from MRO datasets [8] permits the monitoring of long-term surface changes with CaSSIS from as early as MY28 to present. This work assesses the change detection capabilities of CaSSIS by using a combination of simulated and actual CaSSIS images of one of the most active Martian gully sites to date - Gasa Crater [9-12] .

Methods: We initially restricted the simulated CaSSIS images for this study to pre-2012 acquisitions, as our ability to fully photometrically correct CRISM targeted observations for alongtrack variations in emission/phase is confounded due to the loss of the full gimbal range of CRISM in late 2012 [8]. Three coordinated CRISM/CTX pairs were selected for production into simulated CaSSIS cubes based on a combination of favourable geometries, coverage, estimated atmospheric 
dust opacities and notable changes. Simulated CaSSIS images were generated using the procedures in [8] where spectrally and spatially resampled CaSSIS-compatible CRISM and CTX products are combined into a rigorous fully-simulated CaSSIS image using a Gram-Schmidt spectral pansharpening algorithm, which retains I/F information and minimises colour/spectral distortions [13]. To reduce atmospheric contributions, a dark-object-subtraction technique [14] was applied to both simulated and actual images. All images, including the first actual CaSSIS image acquired on Ls 350, MY34 were overlain and compared to one another to identify visible colour and/or morphologic changes. Notable changes were then compared with previously documented activity.

Results and Discussion: We observe 28 possible changes between the simulated and actual CaSSIS image cubes spanning MY28 to MY34 (Fig. 1). Of these, 20 are previously undocumented changes, including 8 putative new changes and 12 fading flows (black-arrows in Fig. 1). All new/previously unrecognized changes are currently under active investigation with associated HiRISE coverage to verify if observed physical changes are not a manifestation of variable illumination conditions. Prominent changes previously observed between MY28 to 30 by $[9,10]$ are all on the northern and north-eastern crater walls (red-arrows in Figs. 1a-c). While one prominent physical change (orange-arrow in Fig. 1c) was previously identified with a simulated CaSSIS image by $[8]$, we note that 5 meter-scale physical changes noted by $[9,10]$ are unresolved by CaSSIS products.
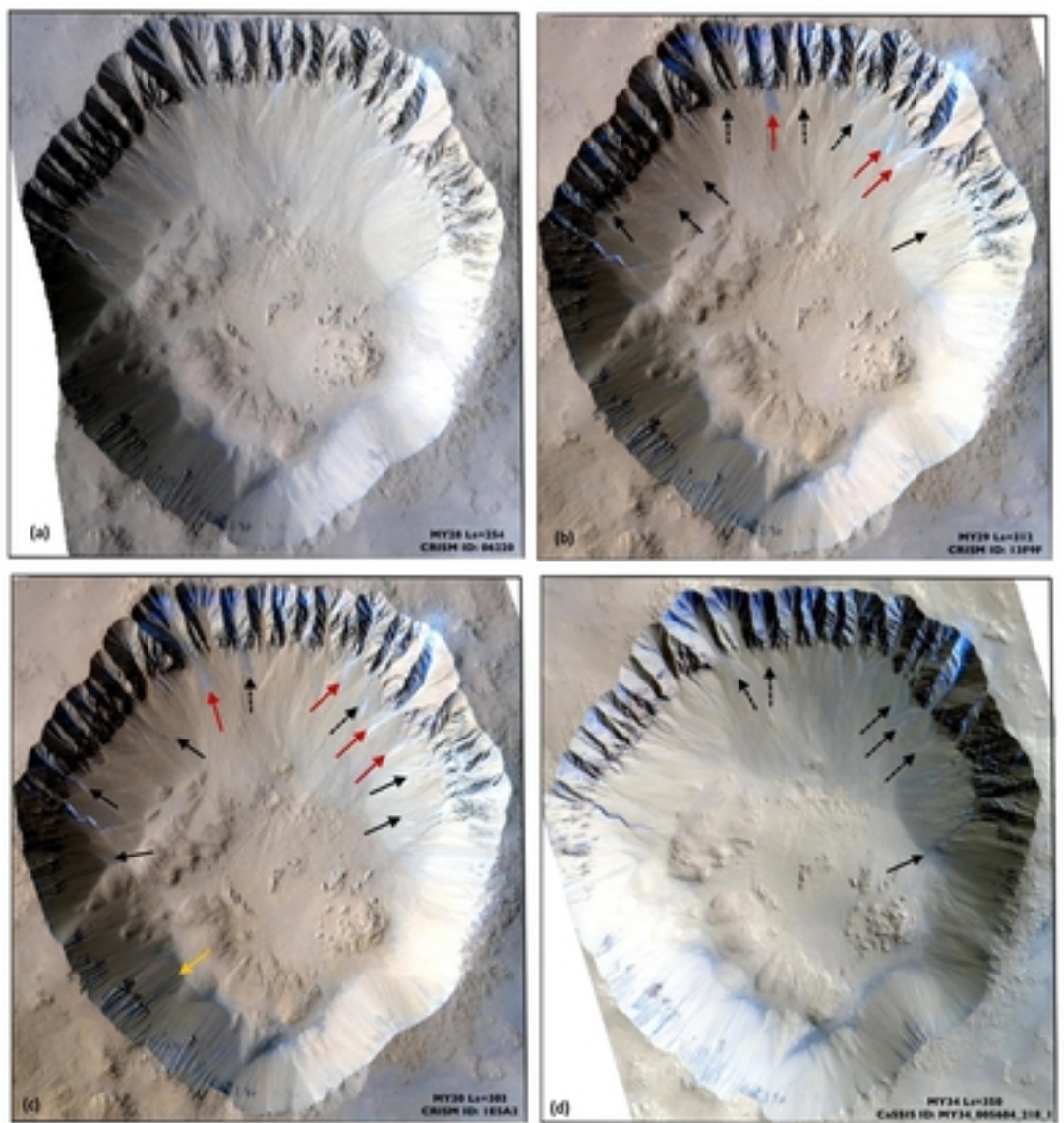

Figure 1: A comparison of three fully-simulated and one actual Cassis infrared colour images (NPB) that show changes that have occurred between 2007 (a), 2009 (b), 2011 (c) and 2019 (d). Red arrows indicate changes documented in Dundas et al. $(2010,2012)$, Orange arrows indicate new changes documented in Tornabene et al. (2018) and Black arrows indicate new, previously undocumented changes observed as a result of using a combination of simulated and actual Cassis images. Bold arrows represent newer or increasing gully activity with respect to the earlier image whereas dashed arrows represent decreasing change/reduction in gully activity with respect to the earlier image.

Putative new colour changes are observed between MY29 and MY34 (Figs. 1, 2). Six of these were 
not readily visible with HiRISE due to lack of colour coverage. One prominent example includes a bright-bluish deposit in the eastern part of the crater (Figs. 1b-c). These deposits have an NIRsignature that suggests they are possible ferrous-bearing materials sourced from the gully alcoves $[8,12,15]$.

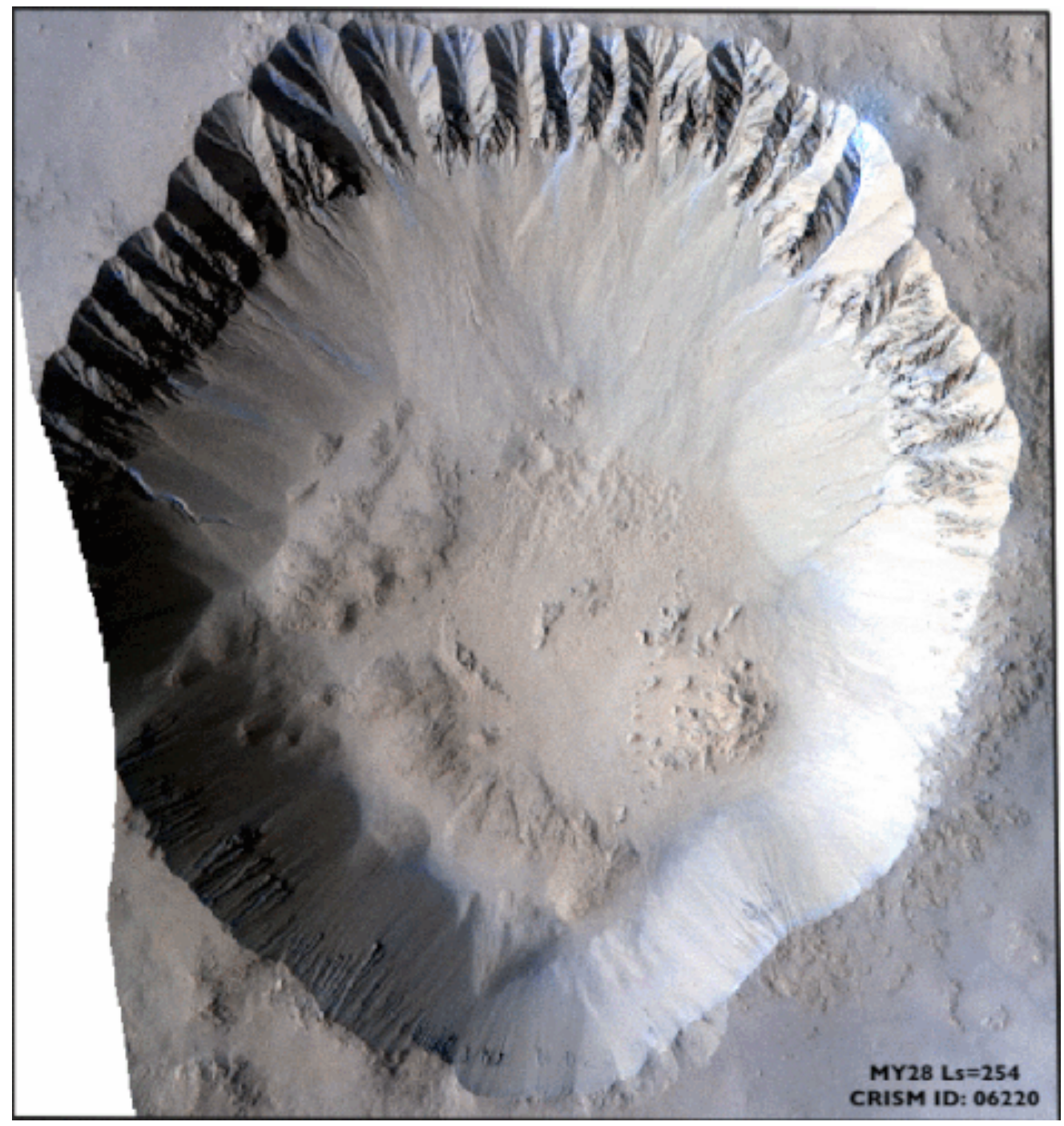

The most-notable recent putative change based on our first CaSSIS acquisition of Gasa (Ls 350, MY34) shows a bright-bluish deposit on the eastern crater wall that spills partially onto the crater floor (Figs. 1d, 2). Although previous HiRISE acquisitions between MY31 and MY34 seem to show possible morphological changes, lack of HiRISE colour coverage over this deposit makes it difficult to verify the activity. A new optimally-positioned HiRISE acquisition later this year will enable us to verify this surface change (if it has not since faded).

Conclusions: This study demonstrates how both simulated and actual CaSSIS cubes are useful for detecting both previously documented and potentially new gully activity at Gasa Crater. While CaSSIS may not capture all small meter-scale physical changes that HiRISE does, it allows for a much-improved colour-change capability over HiRISE. However, continuing to monitor with both instruments is pivotal, as CaSSIS detections of prominent colour changes can be used to reposition HiRISE to better target colour-coverage to validate and characterise meter-scale surface changes. Despite anticipated photometric complications that post-2012 CRISM targeted observations offer, future work will also include an assessment of simulated CaSSIS products generated with post-2012 CRISM and CTX coordinated data to assess identification of both new and previously documented 
HiRISE changes [16] after MY30.

References: [1] McEwen et al. (2010), Icarus 205(1) [2] Bridges et al. (2013), Aeol. Res. 9 [3] Munaretto et al. (2020), PSS 187 [4] Greeley and Iversen (1985), Cambridge Univ. Press [5] McEwen et al. (2007), JGR 112 [6] Malin et al. (2007), JGR 112 [7] Thomas et al. (2017), SSR 212 [8] Tornabene et al. (2018), SSR 214(18) [9] Dundas et al. (2010), GRL 37 [10] Dundas et al. (2012), Icarus 220 [11] Dundas et al. (2015), Icarus 251 [12] Harrison et al. (2019), Geol. Soc. 467(1) [13] Laben et al. (2000), US Patent No: 6011875 [14] Chavez (1988), RSE 24(3) [15] Okubo et al. (2011), Icarus 211(1) [16] Dundas et al. (2019), Geol. Soc. 467(1).

Acknowledgements: We thank the spacecraft and instrument engineering teams for the successful completion of the instrument. CaSSIS is a project of the University of Bern and funded through the Swiss Space Office via ESA's PRODEX programme. The instrument hardware development was also supported by the Italian Space Agency (ASI), INAF/Astronomical Observatory of Padova, and the Space Research Center (CBK) in Warsaw. Support from SGF (Budapest), the University of Arizona (Lunar and Planetary Lab.) and NASA are also gratefully acknowledged. The lead author acknowledges support from Tornabene's funding from the CSA's Planetary and Astronomy Missions Co-Investigator programme (19PACOI07) and the Canadian NSERC Discovery Grant programme (06418-2020 RGPIN). 